\section{Achillessehnenruptur: Nach Naht entwickelt die Wade mehr Kraft}

Die optimale Versorgung einer rupturierten Achillessehne wird nach wie vor kontrovers diskutiert. Bisherige Studien zeigen nach operativer und konservativer Behandlung annähernd gleich gute funktionelle Ergebnisse, wobei die Achillessehnennaht eine aggressivere Rehabilitation ermöglicht. Inwieweit die Operation einen Vorteil im Hinblick auf die wiedergewonnene Kraft im Unterschenkel bietet, war bislang nicht ausreichend belegt.

D as Team um Iikka Lantto von der Universität Oulu hat 32 operativ und 28 konservativ versorgte Patienten mit Achillessehnenruptur in einer randomisierten Studie gegenübergestellt. Als primärer Endpunkt diente der Leppilahti-Score, in den sowohl subjektive Faktoren (Schmerz, Steifheit, Muskelschwäche und Einschränkungen beim Tragen von Schuhen) als auch objektive Kriterien (aktive Beweglichkeit im Knöchel und isokinetische Wadenmuskelkraft) einfließen. Das Optimum liegt bei 100 Punkten, wobei Werte über 90 als exzellentes Ergebnis gelten. 75-89 Punkte stehen für ein gutes, 60-74 für ein akzeptables und weniger als 60 Punkte für ein unzureichendes Resultat.

Die konservativ behandelten Patienten erhielten zunächst für eine Woche einen immobilisierenden Gips, danach für sechs Wochen eine funktionelle Or- these und die Erlaubnis zur Vollbelastung. Nach der fünften Woche war die aktive Plantarflexion erlaubt.

In der Operationsgruppe wurde der Eingriff (Naht nach Krackow) binnen sieben Tagen nach dem Unfall durchgeführt. Anschließend wurde der operierte Fuß in Spitzfußstellung für eine Woche eingegipst. Das weitere Vorgehen entsprach dem in der konservativ behandelten Gruppe.

Die empfohlenen RehabilitationsMaßnahmen waren für alle Patienten gleich; sie umfassten Übungen zur Steigerung der Beweglichkeit im Knöchel, zur Stärkung der Wadenmuskulatur und zur Dehnung der Achillessehne. Ferner bekamen die Patienten Ferseneinlagen verschrieben und wurden zu geeigneten Sportarten beraten. Mit dem Joggen durften die Teilnehmer nach drei Monaten beginnen, mit Impact-Sportar-

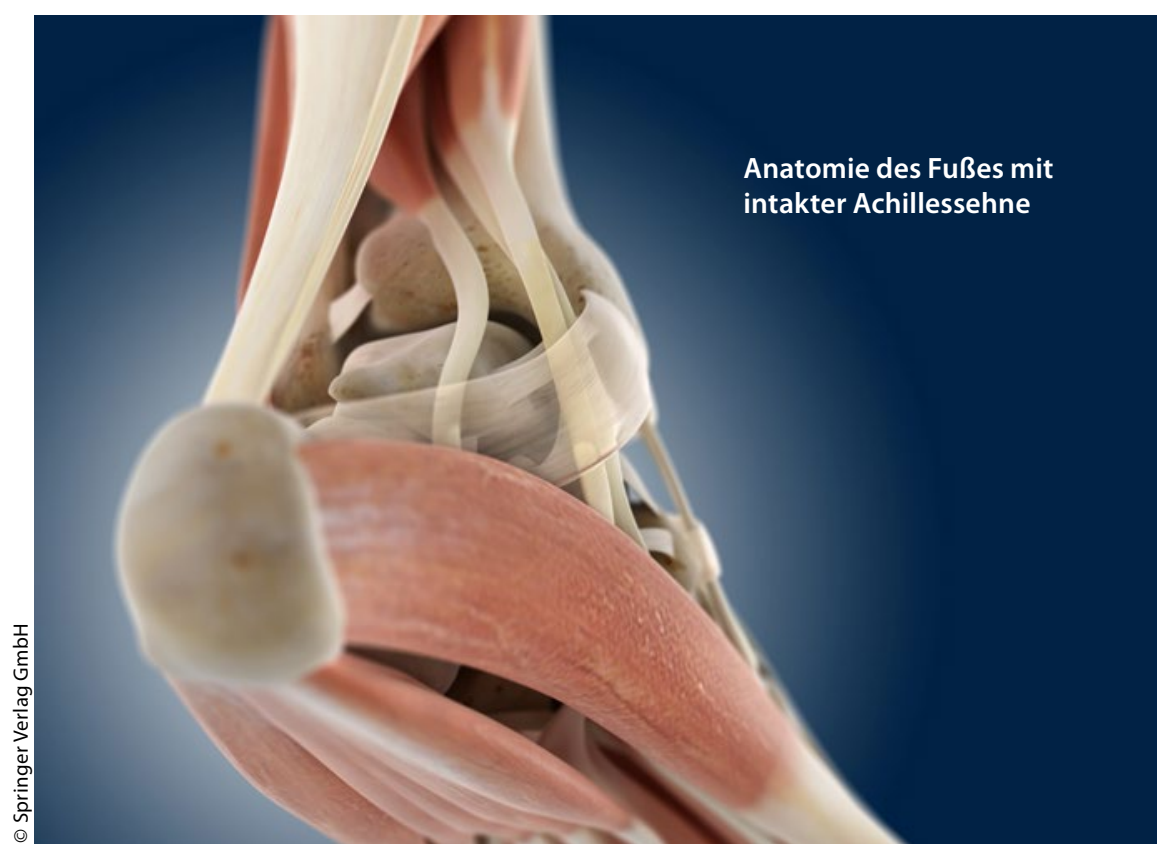

ten (Ballspiele, Kontaktsportarten) nach sechs Monaten.

Nach 18 Monaten betrug der Leppilahti-Score bei den Patienten mit genähter Sehne im Schnitt 79,5 Punkte, bei den konservativ versorgten Patienten 75,7. Der Unterschied ist nicht signifikant. $28 \%$ der operierten Patienten hatten ein exzellentes, $38 \%$ ein gutes und $34 \%$ ein akzeptables Ergebnis. Bei den rein konservativ Behandelten waren es 14,54 und $21 \%$; bei $11 \%$ war das Resultat unbefriedigend.

Deutliche Unterschiede ergaben sich jedoch bei der isolierten Messung der isokinetischen Wadenmuskelkraft. Hier erzielten die operierten Patienten nach sechs Monaten im Schnitt ein bis zu 24\% besseres Ergebnis (um 14,8 Newtonmeter höheres Spitzen-Drehmoment, gemessen mit einem isokinetischen Dynamometer). Nach 18 Monaten betrug der Kraftunterschied noch bis zu $18 \%$. Allerdings ließ sich mit keiner der beiden Vorgehensweisen die Muskelkraft im verletzten Bein wieder auf das Niveau der gesunden Extremität bringen.

$\mathrm{Zu}$ einer erneuten Ruptur der Achillessehne kam es bei $3 \%$ der operierten und bei $14 \%$ der nicht operierten Patienten. Ein Patient in der Op.-Gruppe entwickelte eine tiefe Wundinfektion.

Im RAND-36-Score schnitt die operierte Gruppe deutlich besser ab. Die Vorteile lagen vor allem bei den Parametern „körperliche Funktion“ und "Schmerzen“; dies legt nach Lantto und Kollegen eine bessere Lebensqualität nach der Operation nahe.

Fazit: Die Studie von Lantto et al. hat gezeigt, dass ein Achillessehnenriss nicht unbedingt genäht werden muss. Die Muskelkraft in der Wade lässt sich aber möglicherweise durch die Operation rascher und besser wiederherstellen. Dies sollte man bei der Therapieentscheidung vor allem bei sportlich aktiven Patienten mit höheren Ansprüchen an die eigene Leistungsfähigkeit bedenken, raten die Autoren.

\section{Dr. Elke Oberhofer}

Lantto likka et al. A Prospective Randomized Comparing Surgical and Nonsurgical Treatments of Acute Achilles Tendon Ruptures. Am J Sports Med 2016;

doi: $10.1177 / 0363546516651060$ 